\title{
Enhanced Recovery After Surgery and Radical Cystectomy: A Systematic Review and Meta-Analysis
}

\author{
Muaz Peerbocus' \\ Zeng-Jun Wang ${ }^{2}$ \\ 'Department of Urology, Nanjing Medical \\ University, Nanjing, People's Republic of \\ China; ${ }^{2}$ Department of Urology, The First \\ Affiliated Hospital of Nanjing Medical \\ University, Nanjing, People's Republic of \\ China
}

\begin{abstract}
Introduction:: Enhanced recovery after surgery (ERAS) protocols aim to optimize patient recovery after major surgery. Our study was to examine the evidence of the effectiveness of interventions designed to improve patient outcomes after radical cystectomy.

Design: Systematic review and meta-analysis.

Data Sources: PubMed, Medline, Embase, Cochrane from January 2005 to January 2021 without language restrictions.

Eligibility Criteria: Randomized and non-randomized controlled studies implementing ERAS measuring its interventions on rates of postoperative complications, 30-day readmission, length of stay (LOS) and bowel function after radical cystectomy.

Data Extraction and Synthesis: Two members of the investigating team independently selected studies and evaluated bias using the Cochrane collaboration tool. Meta-analysis of all comparative studies used inversed-weighted, fixed- effects models and random effects models to pool results. Publication bias was graphically assessed using contour-enhanced funnel plots and the Egger's test of funnel plot symmetry.

Results: Fifteen studies were included in our meta-analysis; we observed that ERAS decreased the time for the first bowel movement (standardized mean difference [SMD]: $1.30,95 \% \mathrm{CI}-1.90$ to $-0.70, \mathrm{P}<0.00001)$ and shortened the length of stay (LOS) ([SMD]: $0.49,95 \% \mathrm{CI}-0.77$ to $-0.20,(\mathrm{P}<0.00001)$ ); however, 30 -day readmission (risk ratio [RR]: $0.97,95 \%[\mathrm{CI}] 0.73$ to $1.28, \mathrm{P}=0.52$ ) and the overall postoperative complication rate (risk ratio [RR]: $0.98,95 \%$ confidence interval $[\mathrm{CI}]: 0.88$ to $1.09, \mathrm{P}=0.41)$ showed no significant difference.
\end{abstract}

Keywords: enhanced recovery after surgery, radical cystectomy, length of stay, postoperative complications

\section{Introduction}

Bladder cancer has a high incidence and is associated with high morbidity and mortality. ${ }^{1}$ Radical cystectomy (RC) is the gold standard treatment for high-risk grade non-muscle invasive bladder tumor and muscle invasive bladder cancer (MIBC). ${ }^{2} \mathrm{RC}$ is a complex urological procedure and is associated with poor recovery, often requiring readmission and lengthy hospital stay. Complications are attributed to the patient population who are generally the elderly presenting with comorbidities (eg, coronary artery disease, atherosclerosis, and cerebrovascular accidents). In the past decades, the perioperative management of $\mathrm{RC}$ patients has evolved. In the conventional care, some patients were led down a course of interventions that were based on surgical or anesthesia dogma rather than evidencebased studies. Different routines of care such as bowel preparations and
Correspondence: Zeng-Jun Wang Department of Urology, The First Affiliated Hospital of Nanjing Medical University, 300 Guangzhou Road, Nanjing, People's Republic of China Email zengjunwang2002@sina.com 
preoperative fasting often increase the patient's physical and emotional stress. The systemic release of stress hormones and inflammatory mediators by the central nervous system can be detrimental after major operations, leading to rapid deconditioning and suboptimal outcomes of patients. ${ }^{3}$ There is no single element by itself to counteract this physiological cascade but rather a series of care pathways to help minimalize this response subsequently improving the outcome of the surgery. In the late 1990s, the concept of ERAS was first introduced under the name of fast track recovery to study the effect of the surgical stress response on open colorectal surgery. ${ }^{4}$ It was in 2000 in Denmark that the idea was coined into ERAS evidenced-based perioperative clinical pathways that consider the factors contributing to morbidity and proactively apply measures to reduce or eliminate them. ${ }^{5}$ The application of evidence-based medicine has helped halve the rate of postoperative complications and reduce LOS. ${ }^{6}$ In recent years, many studies have recognized and approved the benefits of ERAS, and it began gaining traction in other surgical specialties such as pancreatic, gynecology and urology. ${ }^{7}$ ERAS pathways for cystectomy patients have tremendous clinical value and several studies have been published on the matter; however, noticeable differences exist in the effect of ERAS protocols and perioperative outcomes. ${ }^{8-21}$

Considering the lack of consistency in study results, as well as the absence of experimental data from high-quality randomized controlled trials (RCT), we conducted a systematic review and meta-analysis to determine the clinical effectiveness of ERAS pathways versus standard care on various perioperative outcomes of interest after RC.

\section{Methods}

\section{Study Selection}

In accordance with the PRISMA guidelines 2020 and Cochrane handbook for systemic reviews of interventions, ${ }^{22,23}$ we conducted a systematic literature search from January 2005 to January 2021 based on databases including PubMed, Medline, Embase and the Cochrane Library without language restrictions. Search terms or keywords used included bladder cancer, enhanced recovery after surgery and radical cystectomy. The following Medical Subject Headings (MESH) terms were used: "Enhanced Recovery After Surgery" [Mesh] OR "Postoperative Care" [Mesh] OR "Recovery of Function" [Mesh] OR "Enhanced Recovery" [tw] OR "fast track protocol*" [tw] OR "eras protocol*" [tw] AND "Cystectomy" [Mesh] OR "Urinary Bladder Neoplasms" [Mesh] OR "Cystectomy" [tw] OR "Bladder tumor*” [tw] OR "bladder cancer" [tw] OR "Radical cystectomy" [tw] AND "Postoperative Complications"[Mesh] OR "length of stay" [tw] OR "injury" [tw] OR "ileus" [tw] OR "incontinence" [tw] OR "shock" [tw]. We also checked the reference lists of all related articles to ensure literature saturation.

\section{Inclusion Criteria and Exclusion Criteria}

Two members of the investigative team (M.P. and Z.J.W.) independently assessed the eligibility of the articles for inclusion in the study and discussed inconsistencies until consensus was obtained. The PICO method was used to define and search for potentially eligible studies.

- P (population): Patients undergoing RC.

- I (intervention): At least one element of ERAS protocols.

- C (comparison): Standard care/conventional therapy/ Non-ERAS.

- O (outcomes of interest): At least one of the following; LOS, time to passage of first stool, readmission, overall complications; Clavien-Dindo classification and postoperative ileus (POI),

Studies that met one of the following criteria were excluded:

(1) The inclusion criteria were not met or no outcomes of interest were reported.

(2) Duplicate publications, non-comparative studies, case reports, editorial articles and reviews.

\section{Data Extraction}

One reviewer (M.P.) independently screened and extracted data from full-texts, citations, and protocols using standardized data collection forms that contained fields for authors, publication year, country, study design, matching variables (age, gender, body mass index, American society of Anesthesiology score, history of previous surgery, clinical staging, operation type, diversion type, operation time, estimated blood loss), and outcomes of interest. The outcomes of interest were as follows: length of hospital stay, time to first passage of stool, rate of 30-day readmission, and overall complications. The overall incidence of complications was classified within the Clavien-Dindo Classification and postoperative ileus. Disagreements between the two reviewers were resolved through discussion. 


\section{Quality Assessment}

M.P. independently used the Cochrane risk-of-bias tool, RoB tool, in Review Manager software (https://commu nity.cochrane.org/help/tools-and-software/revman-5) to assess the risk of bias of RCTs (Figure 1), and there were concerns about risk of bias for the majority of studies. The domains of assessment included (1) random sequence generation (selection bias); (2) allocation concealment (selection bias); (3) blinding of participants and personnel (performance bias); (4) blinding of outcome assessment (detection bias); (5) incomplete outcome data (attrition bias); (6) selective reporting (reporting bias); (7) other bias (such as funding sources). Z.J.W. independently rated the level of evidence of the included studies using the Oxford Centre for Evidence-Based Medicine. ${ }^{24}$

\section{Statistical Analysis}

For studies that reported continuous data as median and range, we estimated the mean and standard deviation (SD) using the method described by Wan et al. ${ }^{25}$ The continuous variables were described as the difference in mean values and the $95 \%$ confidence interval (CI) between ERAS and Non-ERAS. The dichotomous outcomes were analyzed by calculating the risk ratio (RR). The meta-analysis was performed using the RevMan 5.4 software. ${ }^{26}$ An inverseweighted, fixed-effects meta-analysis was performed. The multiple interventions that constitute ERAS make the assumptions of a fixed-effect meta-analysis (all studies in the meta-analysis share one true effect size across all included studies) unlikely; therefore, the degree of heterogeneity between studies was assessed using the chi-square test $(P<0.10)$ and the $I^{2}$ statistic with values $>50 \%$ regarded as being significant heterogeneity. A randomeffects meta-analysis was used when significant heterogeneity was found between studies. We performed sensitivity analyses on recent publication 2015 or later and overall high-risk bias. To assess for publication bias, we used the RStudio coding software (RStudio Team. (2020). RStudio: Integrated Development Environment for R. Boston) ${ }^{27}$ to create contour-enhanced funnel plots and applied the Egger's test of funnel plot symmetry. ${ }^{28}$

\section{Characteristics of Included Studies}

The database search yielded 317 records after duplication removal; we screened 306 records, from which we retrieved 54 full-text records and included 12 studies. $^{8-10,12-17,20,21,29}$
Moreover, we performed a citation and reference search and included three additional studies. ${ }^{11,18,19}$ Fifteen studies that met the inclusion criteria were identified (Figure 2); only 1 study $^{18}$ was a retrospective RCT and the 14 others were prospective cohort studies. A total of 1853 participants were included, of which 953 were grouped as ERAS and 900 as control. The characteristics of each study are summarized in Table 1. The different ERAS elements between each study are summarized in Table 2 .

\section{Results}

The majority of studies focused on the length of stay and overall complications. Studies were conducted in diverse country settings including the United States (two), Italy (two) and one each in India, South Korea, Greece, Sweden, Belgium, Canada, Denmark, England, Egypt and Romania. One included study was retrospective, ${ }^{18} 13$ was prospective randomized control cohort, ${ }^{8-11,13-17,19-21,29}$ and one1 study was nonrandomized prospective cohort. ${ }^{12}$ Time-to-first defecation was recorded across 10 studies, ${ }^{8-10,14,16,17,19-21}$ and $30 \mathrm{~d}$ readmission rate was assessed from 8 studies. ${ }^{9,11,13-15,17,21,29}$

\section{Length of Stay}

Pooled data analysis from 13 studies, ${ }^{8-12,14,15,17-21,29}$ showed that the ERAS group was associated with shorter length of stay compared to the Non-ERAS group. SMD= $-0.49,95 \%$ CI: -0.77 to -0.20 . Heterogeneity: $\mathrm{Chi}^{2}=$ $78.18, d f=12(\mathrm{P}<0.00001) ; \mathrm{I}^{2}=85 \%$ (Figure 3$)$ the random-effects model was used. Participants $=1471$.

\section{Complications}

The overall complications were defined as high-grade, low-grade (Clavien-Dindo classification) and POI. The overall complication rate across 12 studies ${ }^{9-15,17-21}$ showed that ERAS did not reduce the risk of complications with $44.3 \%$ of patients in the ERAS group against $44.79 \%$ of patients in the Non-ERAS group. Furthermore, pooled data from the fixed-effects model detected no significant difference between the two groups: $\mathrm{RR}=0.98$, 95\% CI: 0.88-1.09, with low heterogeneity observed between studies: $\mathrm{Chi}^{2}=11.37, d f=11(\mathrm{P}=0.41) ; \mathrm{I}^{2}=$ $3 \%$ (Figure 4) participants $=1507$. 


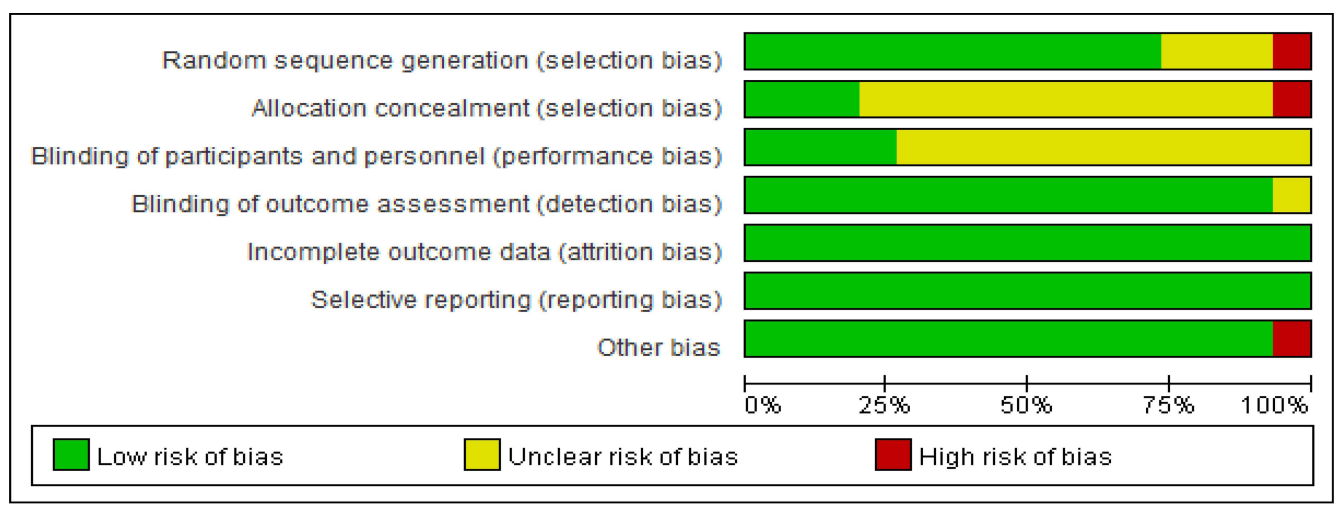

\begin{tabular}{|c|c|c|c|c|c|c|c|}
\hline & 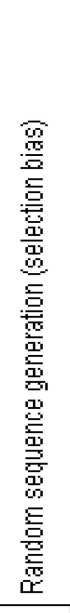 & 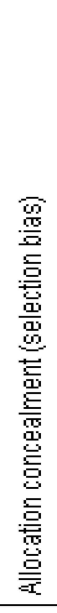 & 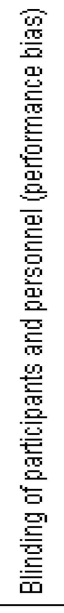 & 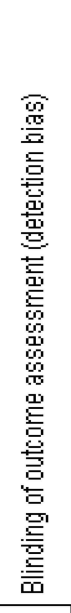 & 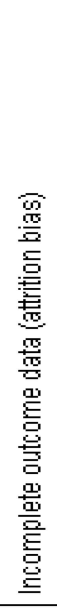 & 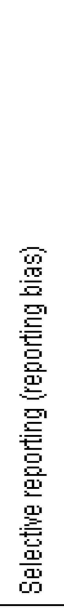 & 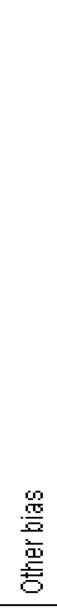 \\
\hline Adamakis 2011 & $\oplus$ & $\theta$ & $?$ & $?$ & $\oplus$ & $\oplus$ & $\oplus$ \\
\hline Bansal 2019 & $\oplus$ & $\oplus$ & $\oplus$ & $\oplus$ & $\oplus$ & $\oplus$ & + \\
\hline Choi 2011 & $\oplus$ & $?$ & $?$ & $\oplus$ & $\oplus$ & $\oplus$ & + \\
\hline Collins 2016 & $\oplus$ & $?$ & $?$ & $\oplus$ & $\oplus$ & $\oplus$ & $\oplus$ \\
\hline Declercq 2015 & $\theta$ & $?$ & $?$ & $\oplus$ & $\oplus$ & $\oplus$ & $\oplus$ \\
\hline Deibert 2016 & $\oplus$ & $?$ & $\oplus$ & $\oplus$ & $\oplus$ & $\oplus$ & + \\
\hline Ercolino 2019 & $?$ & $?$ & $?$ & $\oplus$ & $\oplus$ & $\oplus$ & + \\
\hline Fress 2017 & $?$ & $?$ & $?$ & $\oplus$ & $\oplus$ & $\oplus$ & + \\
\hline Jensen 2015 & $\oplus$ & $\oplus$ & $?$ & $\oplus$ & $\oplus$ & $\odot$ & + \\
\hline Lee CT 2014 & $\oplus$ & $\oplus$ & $?$ & $\oplus$ & $\oplus$ & $\oplus$ & $\theta$ \\
\hline Lin.T 2017 & $\oplus$ & $?$ & $?$ & $\oplus$ & $\oplus$ & $\oplus$ & + \\
\hline Maffezzini 2007 & $?$ & $?$ & $\oplus$ & $\odot$ & $\oplus$ & $\oplus$ & $\oplus$ \\
\hline Mukthar 2013 & $\oplus$ & $?$ & $?$ & $\oplus$ & $\oplus$ & $\oplus$ & + \\
\hline Seham.m 2019 & $\oplus$ & $?$ & $\oplus$ & $\oplus$ & $\oplus$ & $\odot$ & $\oplus$ \\
\hline Vlad 2020 & $\oplus$ & $?$ & $?$ & $\oplus$ & $\oplus$ & $\oplus$ & $\oplus$ \\
\hline
\end{tabular}

Figure I The risk of bias summary of included studies. 


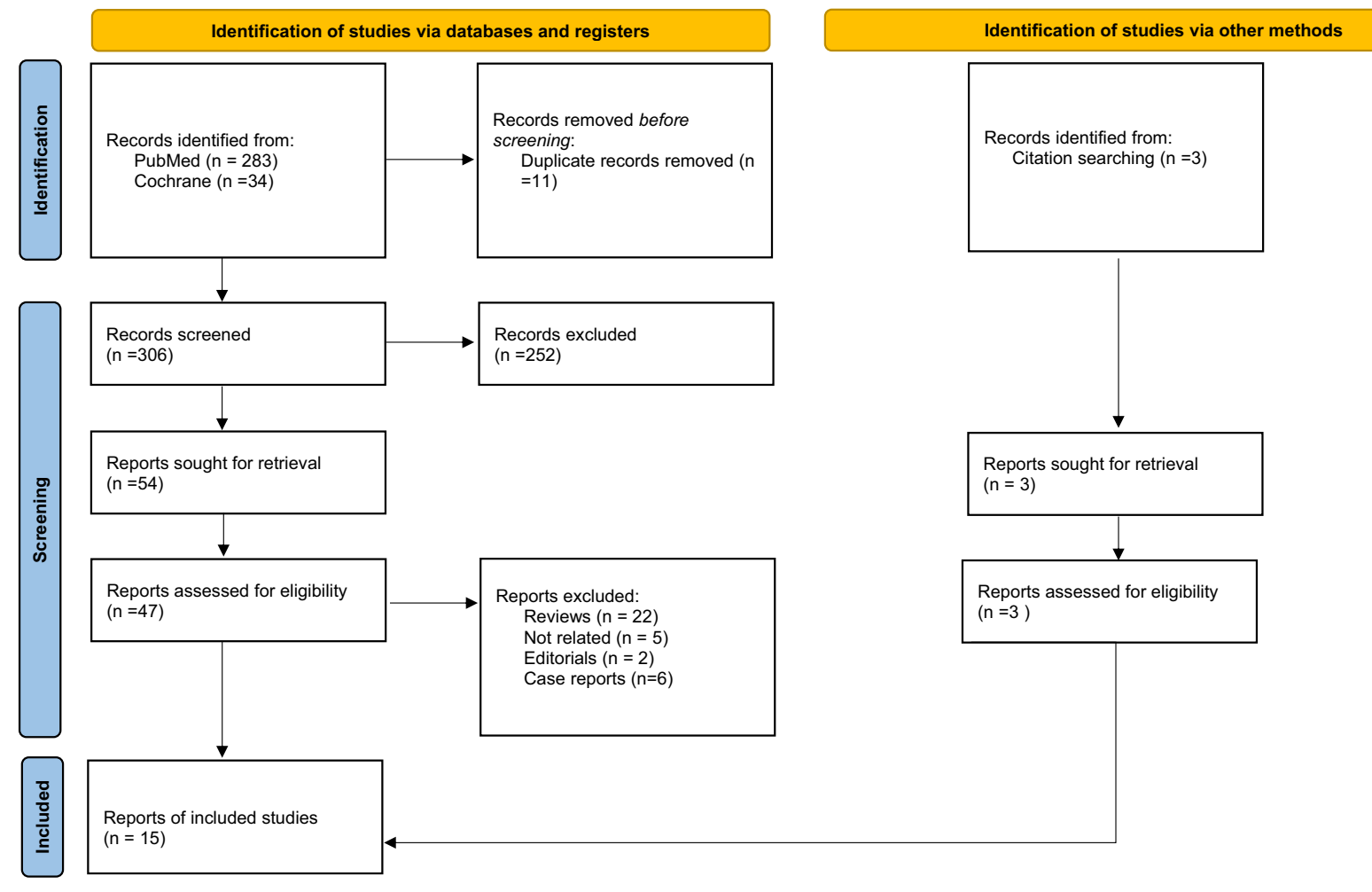

Figure 2 Flowchart of literature selection process.

\section{Bowel Function}

Time-to-first bowel movement was lower in the ERAS group regardless of the diversion type used. Moreover, pooled data from the random-effects model across 10 studies pointed towards a faster return to bowel function in the ERAS group: $\mathrm{SMD}=-1.3095 \%$ but $\mathrm{CI}:-1.90$ to 0.70. Heterogeneity: $\mathrm{Chi}^{2}=185.97, d f=9(\mathrm{P}<0.00001)$; $\mathrm{I}^{2}=95 \%$ (Figure 5) participants $=1216$.

\section{Readmissions}

In general, ERAS did not significantly reduce the probability of patients being readmitted after RC. Moreover, $15.7 \%$ of the patients in the ERAS group were readmitted within $30 \mathrm{~d}$ compared with $15.3 \%$ of the patients in the Non-ERAS group. Pooled data from the fixed-effects model showed no significant difference between the two groups' 30-day readmission rate': $\mathrm{RR}=0.97,95 \% \mathrm{CI}$ : $0.73-1.28$, and there was statistical heterogeneity between studies: $\mathrm{Chi}^{2}=6.14, d f=7(\mathrm{P}=0.52) ; \mathrm{I}^{2}=0 \%$, (Figure 6) participants $=1077$.

\section{Sensitivity Analysis and Publication Bias}

This sensitivity analysis that removes studies with potential bias found no significant changes. To examine small study and publication bias, we used contour-enhanced funnel plots (Figure 7). Visual inspection of the funnel plots indicates possible bias. To further investigate the possibility of bias, we conducted an Egger test for funnel plot asymmetry, and the results are shown in Table 3. With these collective findings, we therefore can conclude that our results are with minimal publication bias.

\section{Discussion}

The study demonstrated that the implementation of ERAS protocols for patients undergoing radical cystectomy quickens the return of bowel function and shortens the length of hospitalization. No significant difference in readmission and complication rates were noted. Urologists have been slow to adopt ERAS despite evidence from colorectal literature showing ERAS protocols lead to improved outcomes. ${ }^{30,31}$ The key principles of the ERAS 
Table I Characteristics of Each Study

\begin{tabular}{|c|c|c|c|c|c|}
\hline First Author, Year & Country & Study Type & ERAS/Non-ERAS & Variable Outcomes & Level of Evidence \\
\hline Bansal et al, ${ }^{9} 2020$ & India & Prospective & $27 / 27$ & {$[1,2,3,4,6,7,9,10]$} & $3 b$ \\
\hline Choi et al, ${ }^{14} 2010$ & South Korea & Prospective & $30 / 30$ & {$[1,2,3,4,6,7,8,9,10]$} & $2 b$ \\
\hline Adamakis et al, ${ }^{8} 2010$ & Greece & Prospective & $22 / 21$ & {$[1,2,3,5,8,9,10]$} & $2 b$ \\
\hline Collins et al, ${ }^{15} 2016$ & Sweden & Prospective & $135 / 86$ & {$[1,2,3,4,5,6,8]$} & $3 b$ \\
\hline Declercq et al, ${ }^{16} 2015$ & Belgium & Prospective & $46 / 48$ & {$[1,2,3,6,7,8,9,10]$} & $3 b$ \\
\hline Deibert et al, ${ }^{17} 2016$ & USA & Prospective & $50 / 52$ & {$[2,4,6,7,8,9,10]$} & $3 b$ \\
\hline Ercolino et al, ${ }^{18} 2019$ & Italy & Prospective & $75 / 116$ & {$[1,2,3,6,7,8,9]$} & $3 b$ \\
\hline Frees et $\mathrm{al}^{29}{ }^{29} 2017$ & Canada & Prospective & $10 / 13$ & {$[1,2,3,4,7,8,9,10]$} & $2 b$ \\
\hline Jensen et al, ${ }^{19} 2014$ & Denmark & Prospective & $50 / 57$ & {$[1,2,3,4,6,7,8,9]$} & $3 b$ \\
\hline Lee et $\mathrm{al}^{20} 2014$ & USA & Prospective & $143 / 137$ & {$[1,2,3,6,7,8,9,10]$} & $3 b$ \\
\hline Lin et al, 2017 & China & Prospective & $144 / 145$ & {$[1,2,3,6,7,8,9,10]$} & $3 b$ \\
\hline Maffezzini et al, ${ }^{10} 2007$ & Italy & Retrospective & $71 / 40$ & {$[1,2,4,6,8,9]$} & $3 b$ \\
\hline Mukhtar et al, ${ }^{\prime \prime} 2013$ & England & Prospective & $51 / 26$ & {$[1,2,3,4,5,6,7,8,9]$} & $3 b$ \\
\hline Moeen et al, ${ }^{12} 2019$ & Egypt & Prospective & $54 / 57$ & {$[1,2,3,4,6,7,8,9,10]$} & $3 b$ \\
\hline Vlad et al, ${ }^{13} 2020$ & Romania & Prospective & $45 / 45$ & {$[1,2,3,4,6,7,8,9,10]$} & $3 b$ \\
\hline
\end{tabular}

Notes: Includes the number of patients in ERAS group against the number of patients in the Non-ERAS group. I) age, 2) gender, 3) body mass index (BMI), 4) American Society of Anesthesiology (ASA) Score, 5) history of previous surgery, 6) clinical stage, 7) operation type, 8) diversion type, 9) operation time, 10) estimated blood loss. Abbreviation: ERAS, enhanced recovery after surgery.

model are evidence-based and include preoperative counseling, preoperative nutrition, omission of mechanical bowel preparation (MBP), limiting preoperative fasting, carbohydrate loading (non-diabetic patients), standardized analgesic regimens, fluid management, prevention of hypothermia and deep venous thrombosis, minimally invasive approach, antimicrobial prophylaxis, prevention of POI, early mobilization and early oral diet. ${ }^{32}$ The standardized pathways are designed to achieve optimum outcome from major surgeries and provide the necessary measures to attain such goals. Two studies did not omit MBP, ${ }^{11,18}$ all studies implemented early mobilization, early removal of NG tube, early oral feeding and pain management. The other pathways varied among studies for instance some studies show that ERAS pathways can shorten LOS, ${ }^{10,11,29}$ whereas others do not; ${ }^{8,15,20}$ most studies highlight the effect of ERAS pathways on reducing time to recovery of bowel function, ${ }^{9,10,14,17,29}$ with the exception of one ${ }^{8}$; some studies demonstrate that ERAS protocols reduce readmission rates, ${ }^{14,21,33}$ yet three studies did not ${ }^{11,15,29}$; some studies concluded that the standardized protocol reduces the overall complications ${ }^{9,12,19-21}$; however some studies showed no change in morbidity. ${ }^{13,14,18}$ The differences in pathways most certainly raise the question about which elements to universally adopt, but the focus of this study was to assess whether their implementations have any clinical impact. Our study showed the importance in adopting the ERAS protocols to improve perioperative outcomes of RC patients compared to a traditional approach and that the multimodal nature of ERAS is better than individually focusing on a single element within it. A recent umbrella review of 23 meta-analyses across multiple surgical specialties including urology (3) by Zhang et $\mathrm{al}^{34}$ showed strong evidence that ERAS pathways can reduce LOS and cost without increasing morbidity and readmission. Despite these positive results, we need to 


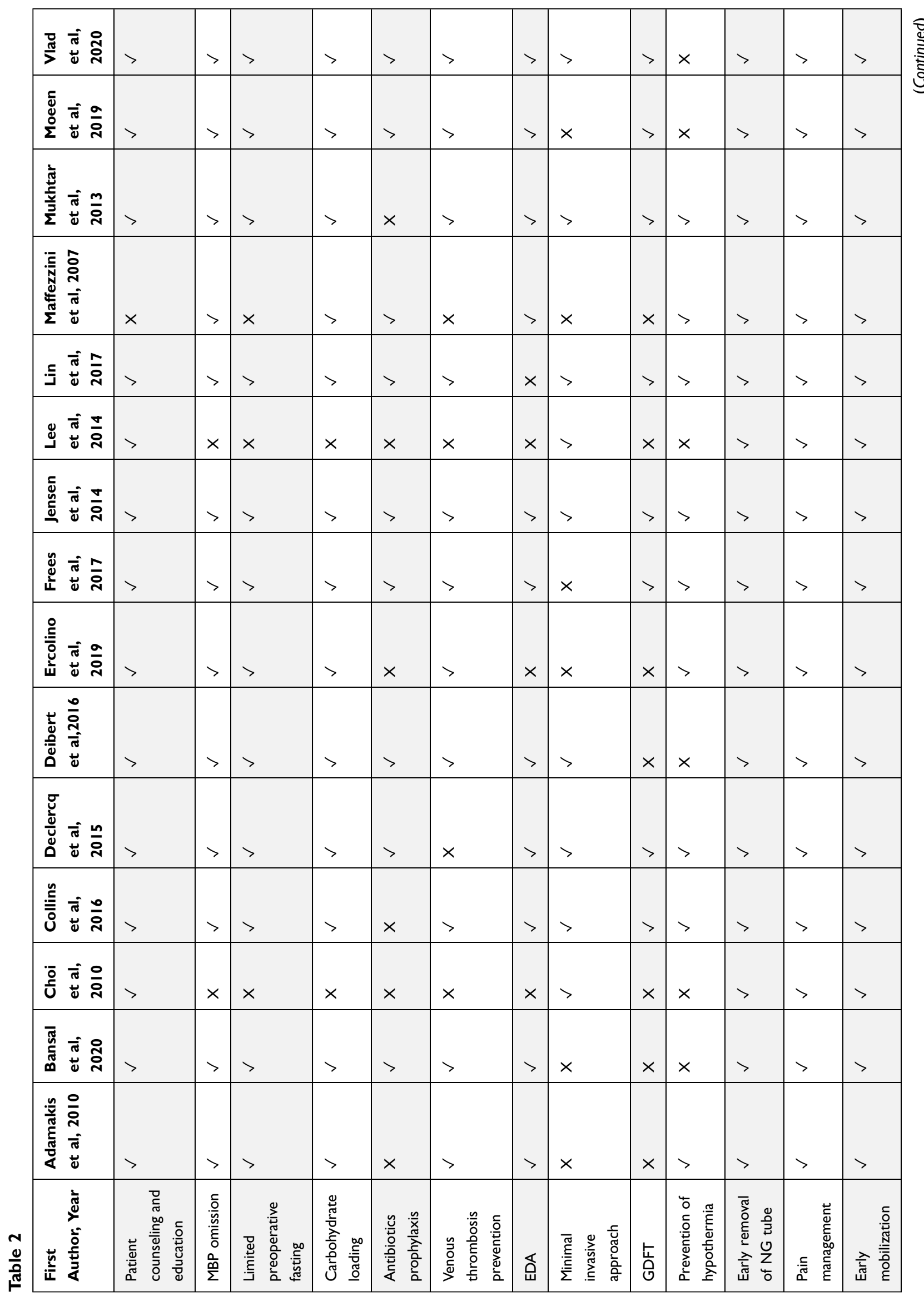




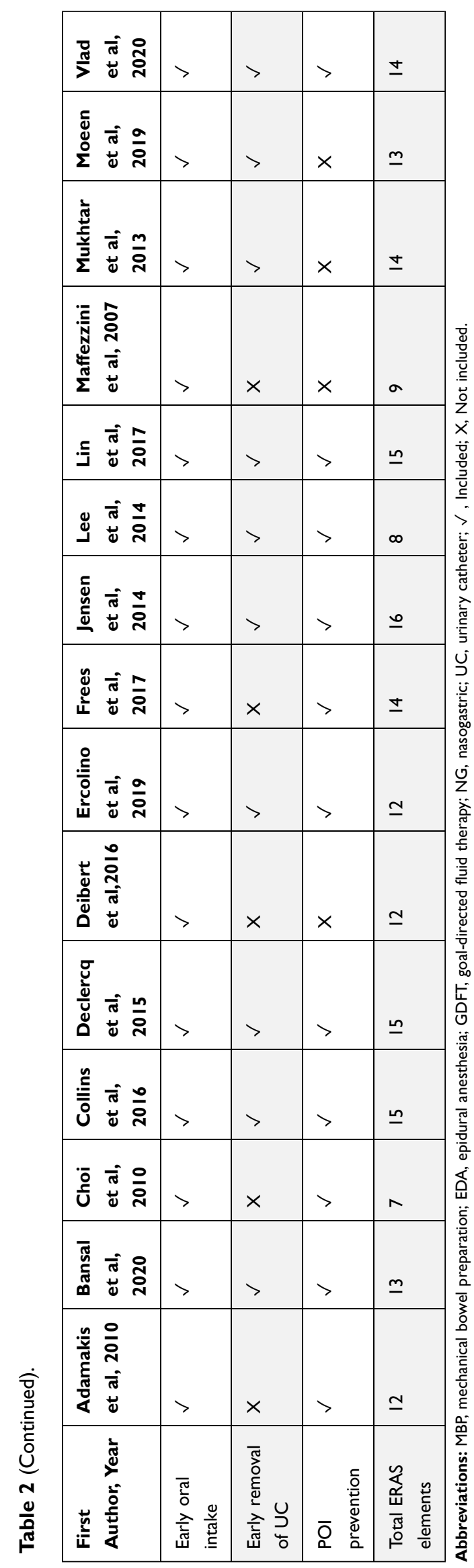




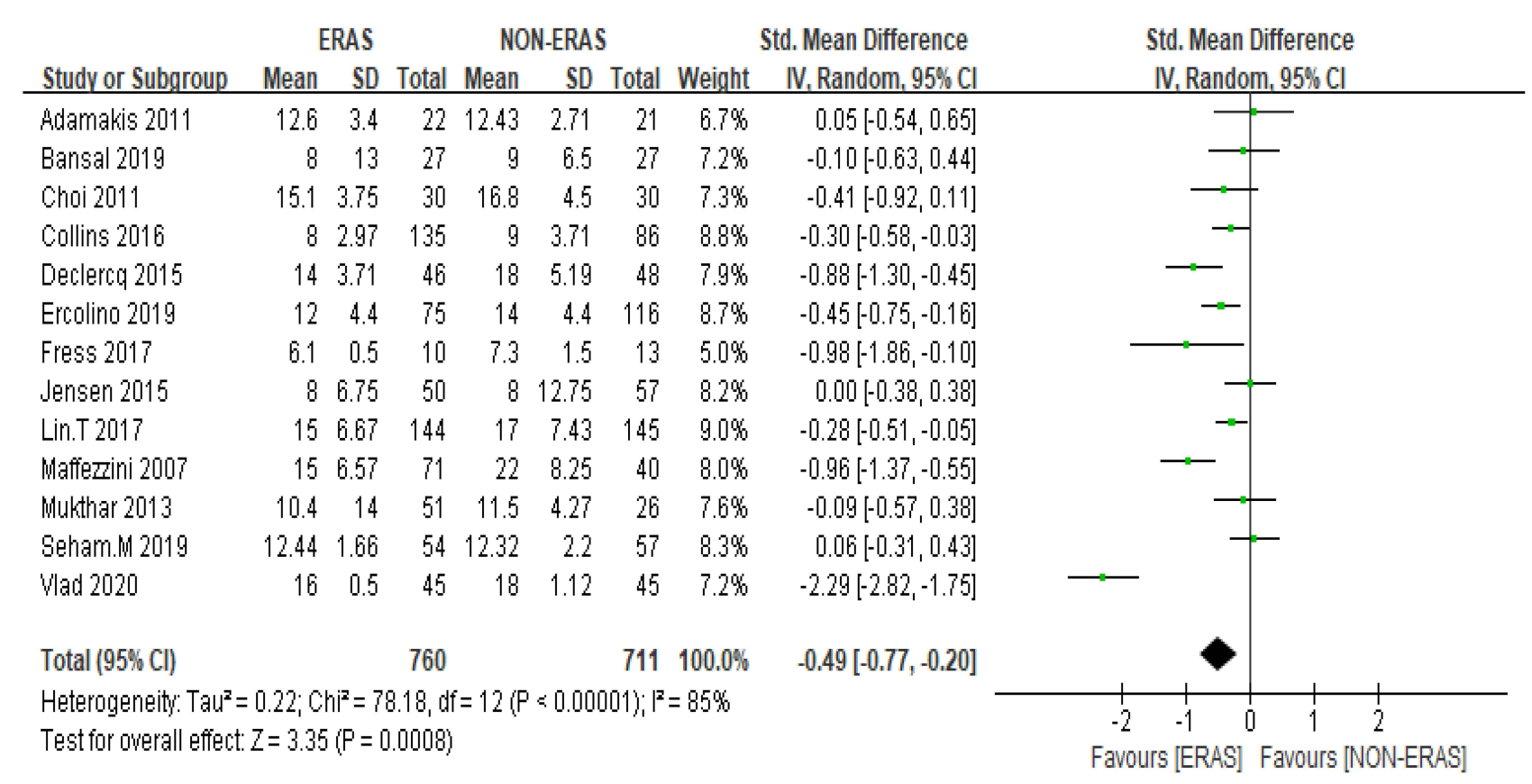

Figure 3 Forest plot displaying a random-effects meta-analysis of the effect of enhanced recovery after surgery (ERAS) on length of stay after cystectomy. Weights are from random-effects analysis.

Abbreviations: $\mathrm{Cl}$, confidence interval; SMD, standardized mean difference.

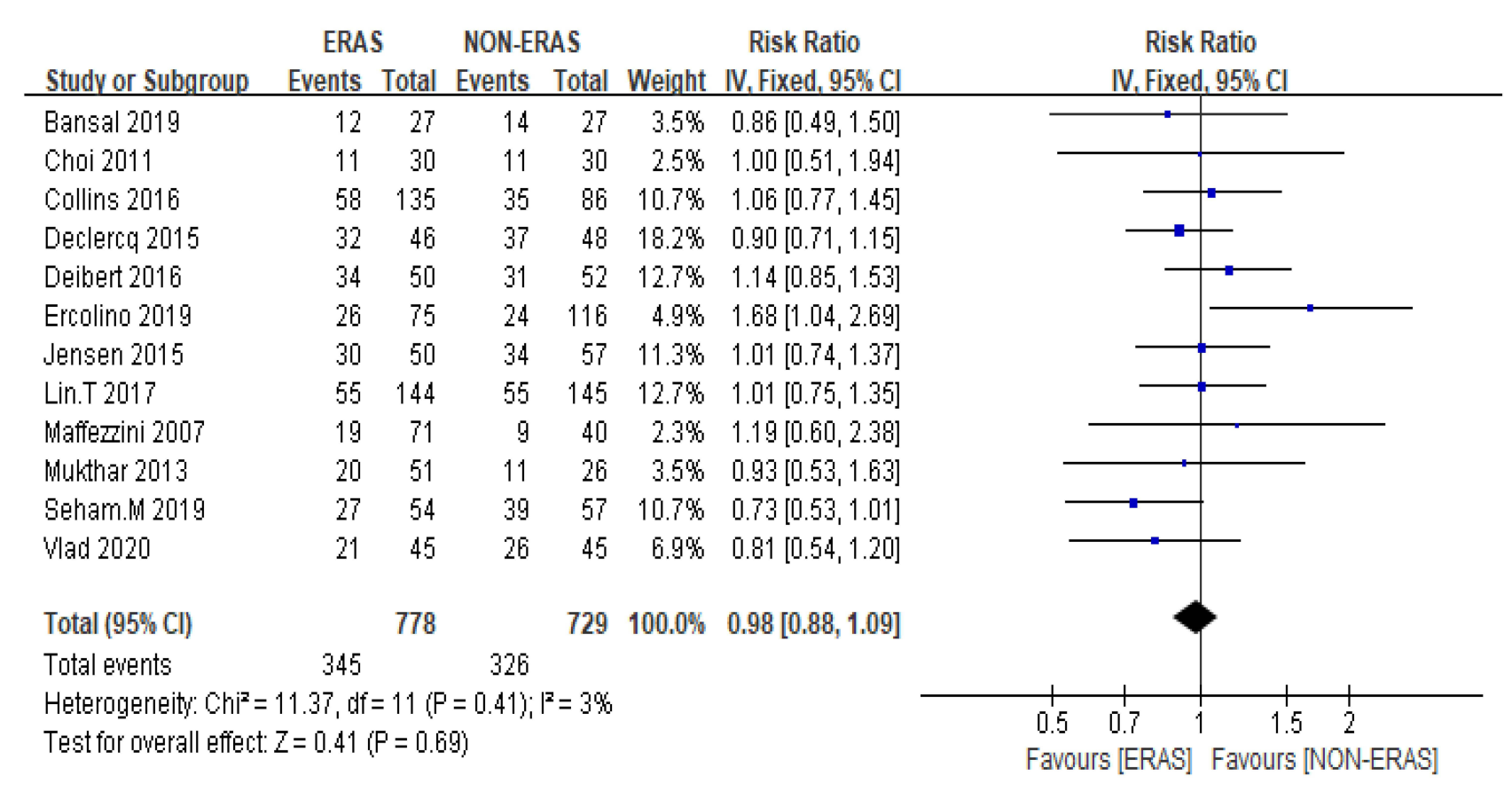

Figure 4 Forest plot displaying a fixed-effects meta-analysis of the effect of enhanced recovery after surgery (ERAS) on complication rates after cystectomy. Abbreviations: $\mathrm{Cl}$, confidence interval; $\mathrm{RR}$, risk ratio. 


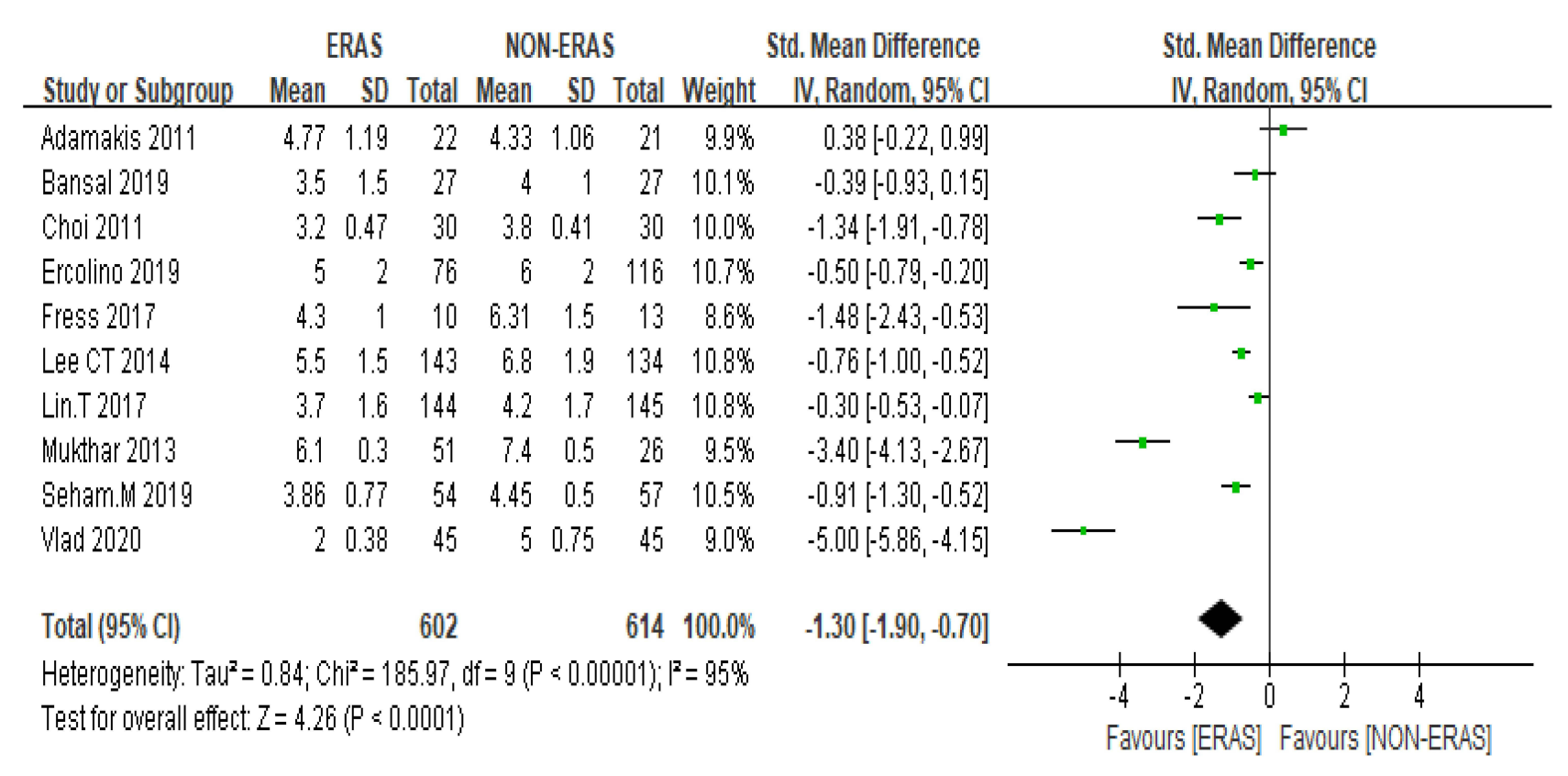

Figure 5 Forest plot displaying a random-effects meta-analysis of the effect of enhanced recovery after surgery (ERAS) on time to bowel function recovery after cystectomy. Weights are from random-effects analysis.

Abbreviations: $\mathrm{Cl}$, confidence interval; SMD, standardized mean difference.

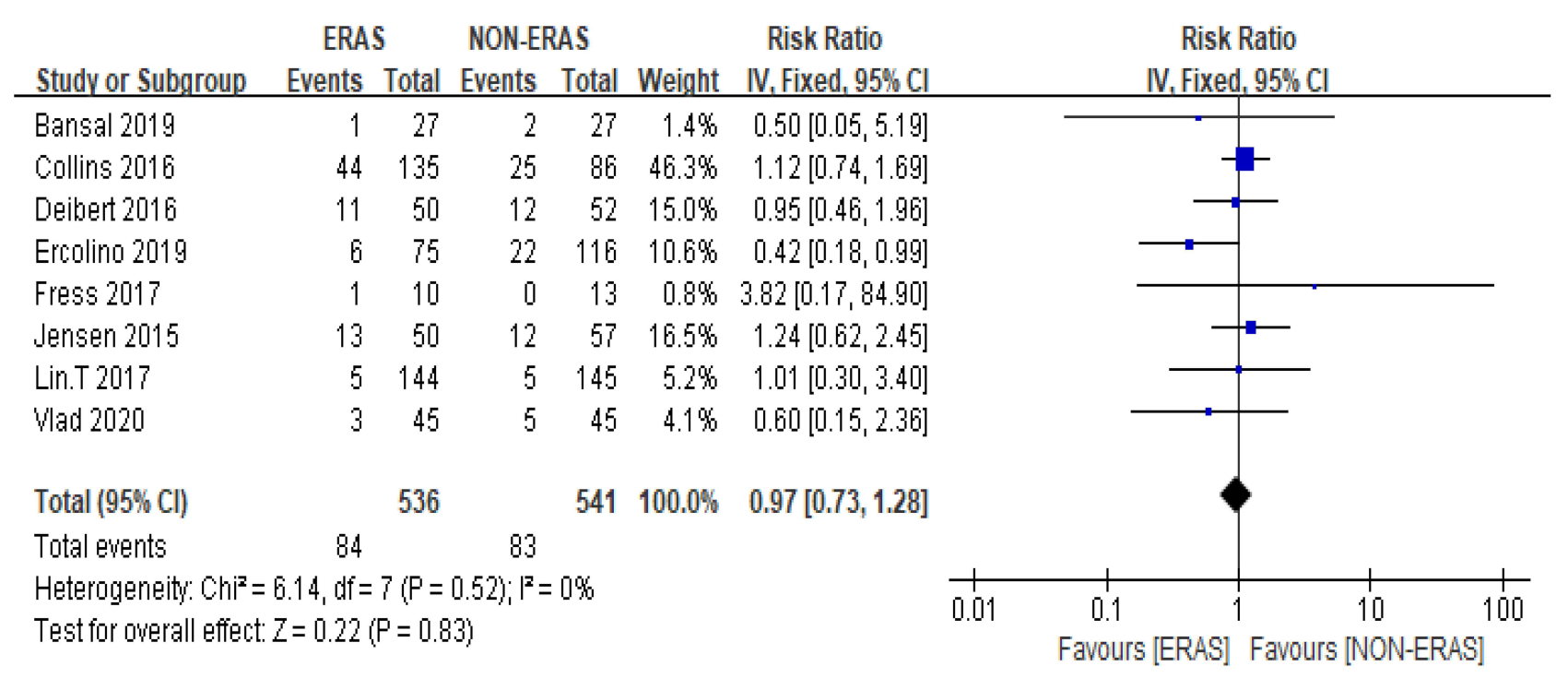

Figure 6 Forest plot displaying a fixed-effects meta-analysis of the effect of enhanced recovery after surgery (ERAS) readmission rates within $30 \mathrm{~d}$ after cystectomy. Abbreviations: $\mathrm{Cl}$, confidence interval; $\mathrm{RR}$, risk ratio.

exercise caution in interpreting these findings, there are limitations that cannot be ignored. The primary limitation is the limited number of RCT studies. Only two studies were blinded, all studies had at least an unclear bias in one domain. Another limitation was that we did not perform a subgroup analysis based on the operation type and diversion type, we grouped all patients implementing the ERAS protocols under one group, which may also introduce 
A

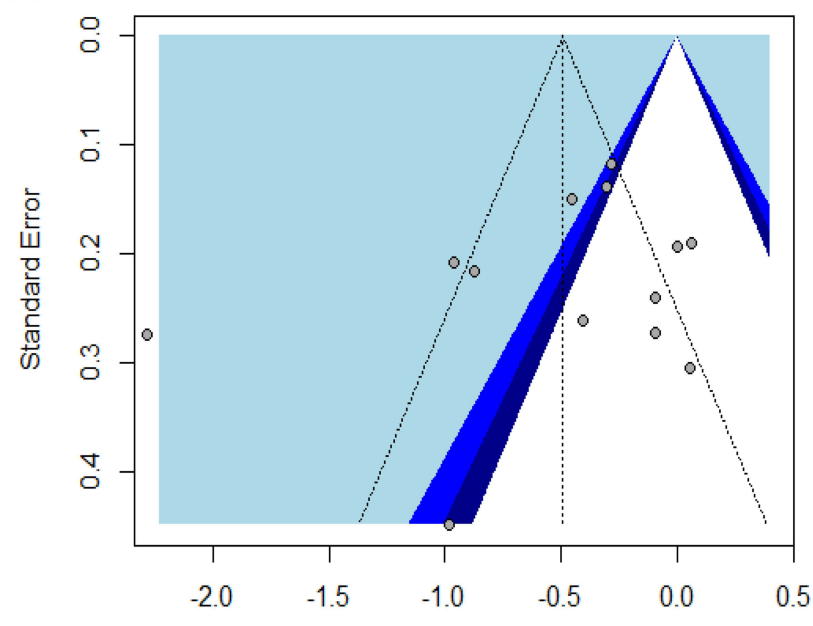

Hedges' g

C

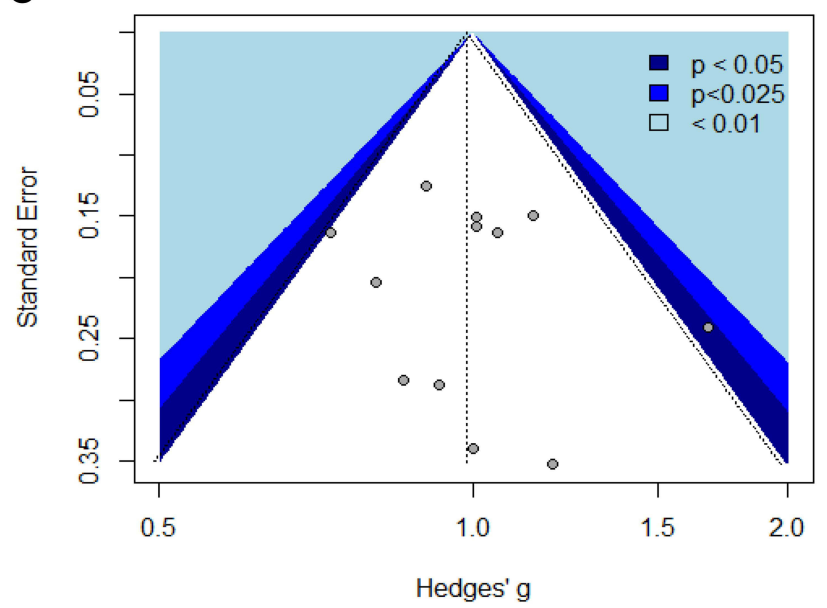

B

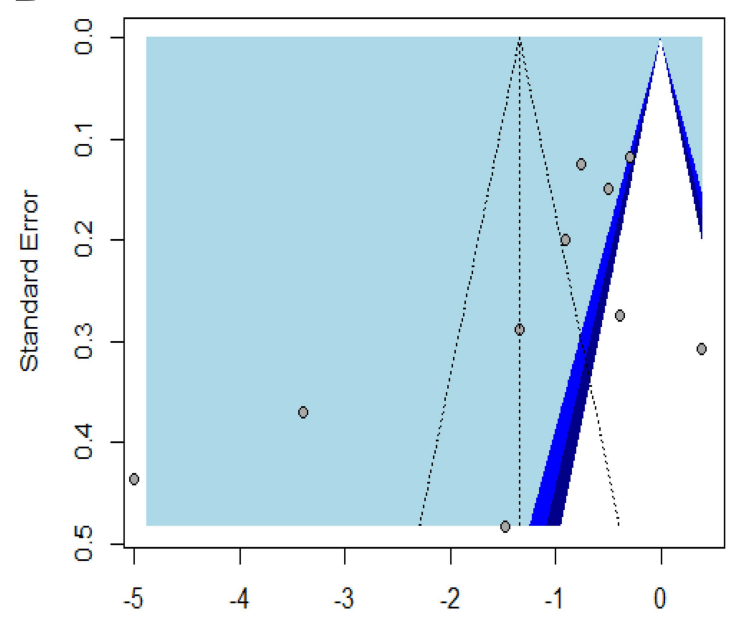

Hedges' g

D

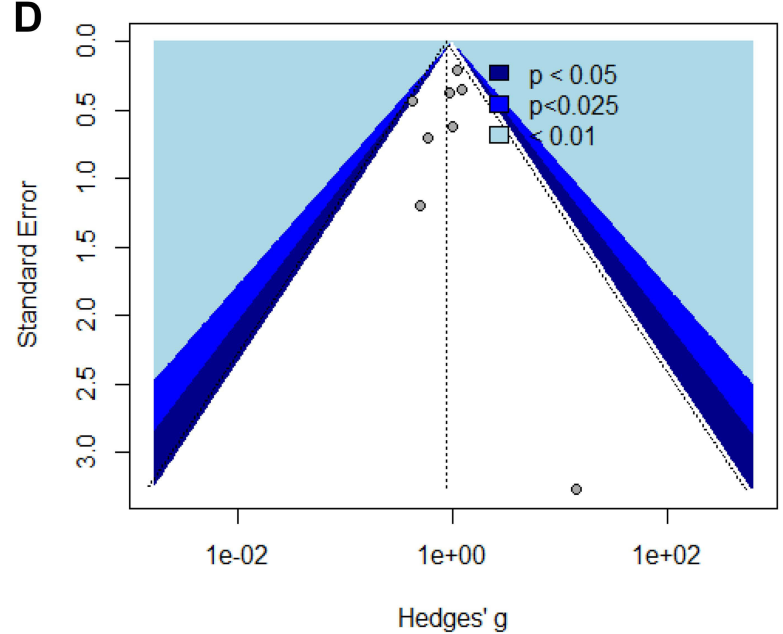

Figure 7 Contour-enhanced funnel plots for (A). Length of stay (B). bowel function (C). rate of complications (D). $30 \mathrm{~d}$ readmission.

biased results. The publication bias was assessed by visual interpretation of funnel plots and Egger's test for funnel plot symmetry. Lastly, health economics and quality of life were not included in our study due to lack of data. Despite these limitations, pooled data displayed the clinical efficacy of ERAS. Our findings indicated that ERAS

Table 3 Eggers' Test

\begin{tabular}{|l|c|c|c|c|}
\hline Outcomes & P-Intercept & 95\% Cl & t value & P value \\
\hline LO.S & -2.143 & {$[-6.43$ to 2.14$]$} & -0.981 & 0.347 \\
Bowel movement & -5.88 & {$[-11.28$ to -0.48$]$} & -2.135 & 0.065 \\
Overall complications & 0.57 & {$[-1.34$ to 2.54$]$} & 0.602 & 0.560 \\
30d-readmission & -0.37 & {$[-1.65$ to 0.92$]$} & -0.564 & 0.593 \\
\hline
\end{tabular}

Abbreviations: $\mathrm{LOS}$, length of stay, $\mathrm{Cl}$, confidence interval. 
protocols on perioperative outcomes of radical cystectomy provide a better improvement of the length of hospital stay and early rehabilitation of gastrointestinal function. Moreover, ERAS protocols did not increase the risk of adverse events, when compared with conventional protocols. These data have important clinical significance and we believe that our study further contributes to the body of evidence that supports the clinical value of ERAS in an effort to improve patients' outcomes in the cystectomy population.

\section{Author Contributions}

Study concept and design: Peerbocus, Wang.

Acquisition of data: Peerbocus.

Analysis and interpretation of data: Peerbocus, Wang.

Critical revision of the manuscript for important intellectual content: Wang.

Statistical analysis: Peerbocus.

Supervision: Wang.

Other: None.

All authors made a significant contribution to the work reported, whether that is in the conception, study design, execution, acquisition of data, analysis and interpretation, or in all these areas; took part in drafting, revising or critically reviewing the article; gave final approval of the version to be published; have agreed on the journal to which the article has been submitted; and agree to be accountable for all aspects of the work.

\section{Funding}

There is no funding to report.

\section{Disclosure}

The authors declare that they have no competing interests.

\section{References}

1. Kirkali Z, Chan T, Manoharan M, et al. Bladder cancer: epidemiology, staging and grading, and diagnosis. Urology. 2005;66(6SUPPL. 1):4-34. doi:10.1016/j.urology.2005.07.062

2. Tan WS, Lamb BW, Kelly JD. Complications of radical cystectomy and orthotopic reconstruction. Adv Urol. 2015;2015:1-7. doi: $10.1155 / 2015 / 323157$

3. Finnerty CC, Mabvuure NT, Kozar RA, Herndon DN. The surgically induced stress response. J Parenter Enter Nutr. 2013;37:21S-29S. doi:10.1177/0148607113496117

4. Kehlet H. Multimodal approach to control postoperative pathophysiology and rehabilitation. Br J Anaesth. 1997;78(5):606-617. doi:10.1093/bja/78.5.606

5. Kehlet H, Wilmore DW. Multimodal strategies to improve surgical outcome. Am J Surg. 2002;183(6):630-641. doi:10.1016/S00029610(02)00866-8
6. Varadhan KK, Neal KR, Dejong CHC, Fearon KCH, Ljungqvist O, Lobo DN. The enhanced recovery after surgery (ERAS) pathway for patients undergoing major elective open colorectal surgery: a meta-analysis of randomized controlled trials. Clin Nutr. 2010;29 (4):434-440. doi:10.1016/j.clnu.2010.01.004

7. Ansari D, Gianotti L, Schröder J, Andersson R. Fast-track surgery: procedure-specific aspects and future direction. Langenbecks Arch Surg. 2013;398(1):29-37. doi:10.1007/s00423-012-1006-9

8. Adamakis I, Tyritzis SI, Koutalellis G, et al. Early removal of nasogastric tube is beneficial for patients undergoing radical cystectomy with urinary diversion. Int Braz J Urol. 2011;37(1):42-48. doi:10.1590/S1677-55382011000100006

9. Bansal D, Nayak B, Singh P, et al. Randomized controlled trial to compare outcomes with and without the enhanced recovery after surgery protocol in patients undergoing radical cystectomy. Indian J Urol. 2020;36(2):95-100. doi:10.4103/iju.IJU_11_20

10. Choi H, Kang SH, Yoon DK, et al. Chewing gum has a stimulatory effect on bowel motility in patients after open or robotic radical cystectomy for bladder cancer: a prospective randomized comparative study. Urology. 2011;77(4):884-890. doi:10.1016/j. urology.2010.06.042

11. Collins JW, Adding C, Hosseini A, et al. Introducing an enhanced recovery programme to an established totally intracorporeal robot-assisted radical cystectomy service. Scand J Urol. 2016;50 (1):39-46. doi:10.3109/21681805.2015.1076514

12. Declercq P, De Win G, Van der Aa F, et al. Reduced length of stay in radical cystectomy patients with oral versus parenteral post-operative nutrition protocol. Int $J$ Clin Pharm. 2015;37(2):379-386. doi:10.1007/s11096-015-0072-9

13. Deibert CM, Silva MV, RoyChoudhury A, et al. A prospective randomized trial of the effects of early enteral feeding after radical cystectomy. Urology. 2016;96:69-73. doi:10.1016/j.urology.2016.06.045

14. Ercolino A, Bianchi FM, Chessa F, et al. Perioperative outcomes of fast track protocol applied to patients treated with radical cystectomy and intestinal urinary diversion: a comparison with standard management in a high-volume center. Eur Urol Suppl. 2019;18(9):e3280e3281. doi:10.1016/s1569-9056(19)33686-3

15. Jensen BT, Petersen AK, Jensen JB, Laustsen S, Borre M. Efficacy of a multiprofessional rehabilitation programme in radical cystectomy pathways: a prospective randomized controlled trial. Scand J Urol. 2015;49(2):133-141. doi:10.3109/21681805.2014.967810

16. Lee CT, Chang SS, Kamat AM, et al. Alvimopan accelerates gastrointestinal recovery after radical cystectomy: a multicenter randomized placebo-controlled trial. Eur Urol. 2014;66(2):265-272. doi:10.1016/j.eururo.2014.02.036

17. Lin T, Li K, Liu H, et al. Enhanced recovery after surgery for radical cystectomy with ileal urinary diversion: a multi-institutional, randomized, controlled trial from the Chinese bladder cancer consortium. World J Urol. 2018;36(1):41-50. doi:10.1007/s00345-017-2108-3

18. Maffezzini M, Gerbi G, Campodonico F, Parodi D. Multimodal perioperative plan for radical cystectomy and intestinal urinary diversion. I. Effect on recovery of intestinal function and occurrence of complications. Urology. 2007;69(6):1107-1111. doi:10.1016/j. urology.2007.02.062

19. Mukhtar S, Ayres BE, Issa R, Swinn MJ, Perry MJA. Challenging boundaries: an enhanced recovery programme for radical cystectomy. Ann $R$ Coll Surg Engl. 2013;95(3):200-206. doi:10.1308/ $003588413 X 13511609957579$

20. Moeen SM, Moeen AM. Usage of intravenous lidocaine infusion with enhanced recovery pathway in patients scheduled for open radical cystectomy: a randomized trial. Pain Physician. 2019;22(2): E71-E80. doi:10.36076/ppj/2019.22.e71

21. Vlad O, Catalin B, Mihai H, et al. Enhanced recovery after surgery (ERAS) protocols in patients undergoing radical cystectomy with ileal urinary diversions: a randomized controlled trial. Medicine. 2020;99(27):e20902. doi:10.1097/MD.0000000000020902 
22. Page MJ, McKenzie JE, Bossuyt PM, et al. The PRISMA 2020 statement: an updated guideline for reporting systematic reviews. BMJ. 2021;372:n71. doi:10.1136/bmj.n71

23. Cochrane Handbook for Systematic Reviews of Interventions. Cochrane training. Available from: https://training.cochrane.org/hand book. Accessed May 2, 2021.

24. Oxford centre for evidence-based medicine: levels of evidence (March 2009) - Centre for Evidence-Based Medicine (CEBM), University of Oxford. Available from: https://www.cebm.ox.ac.uk/ resources/levels-of-evidence/oxford-centre-for-evidence-basedmedicine-levels-of-evidence-march-2009. Accessed May 2, 2021.

25. Wan X, Wang W, Liu J, Tong T. Estimating the sample mean and standard deviation from the sample size, median, range and/or interquartile range. BMC Med Res Methodol. 2014;14(1):1-13. doi:10.1186/1471-2288-14-135

26. RevMan 5 download. Cochrane training. Available from: https://train ing.cochrane.org/online-learning/core-software-cochrane-reviews/rev man/revman-5-download. Accessed May 2, 2021.

27. RStudio. Open source \& professional software for data science teams RStudio. Available from: https:/www.rstudio.com/. Accessed May 4, 2021.

28. Sterne JAC, Harbord RM. Funnel plots in meta-analysis. Stata J Promot Commun Stat Stata. 2004;4(2):127-141. doi:10.1177/ 1536867x0400400204

29. Frees SK, Aning J, Black P, et al. A prospective randomized pilot study evaluating an ERAS protocol versus a standard protocol for patients treated with radical cystectomy and urinary diversion for bladder cancer. World J Urol. 2018;36(2):215-220. doi:10.1007/ s00345-017-2109-2
30. Eskicioglu C, Forbes SS, Aarts MA, Okrainec A, McLeod RS. Enhanced recovery after surgery (ERAS) programs for patients having colorectal surgery: a meta-analysis of randomized trials. $J$ Gastrointest Surg. 2009;13(12):2321-2329. doi:10.1007/s11605009-0927-2

31. Lassen K. Consensus review of optimal perioperative care in colorectal surgery. Arch Surg. 2009;144(10):961. doi:10.1001/archsurg.20 09.170

32. Cerantola Y, Valerio M, Persson B, et al. Guidelines for perioperative care after radical cystectomy for bladder cancer: enhanced recovery after surgery $\left(\right.$ ERAS $\left.^{\circledR}\right)$ society recommendations. Clin Nutr. 2013;32(6):879-887. doi:10.1016/j.clnu.2013. 09.014

33. Nayak B, Bansal D, Singh P, Seth A, Nayyar R, Ramachandran R. Randomized controlled trial to compare the length of stay, perioperative outcomes and complications in patients undergoing radical cystectomy with and without the enhanced recovery after surgery (ERAS) protocol in a tertiary care centre in India. Eur Urol Suppl. 2019;18(1):e1326. doi:10.1016/s1569-9056(19)30957-1

34. Zhang X, Yang J, Chen X, Du L, Li K, Zhou Y. Enhanced recovery after surgery on multiple clinical outcomes: umbrella review of systematic reviews and meta-analyses. Medicine. 2020;99(29): e20983. doi:10.1097/MD.0000000000020983

\section{Publish your work in this journal}

Research and Reports in Urology is an international, peer-reviewed, open access journal publishing original research, reports, editorials, reviews and commentaries on all aspects of adult and pediatric urology in the clinic and laboratory including the following topics: Pathology, pathophysiology of urological disease; Investigation and treatment of urological disease; Pharmacology of drugs used for the treatment of urological disease. The manuscript management system is completely online and includes a very quick and fair peer-review system, which is all easy to use. Visit http://www.dovepress.com/ testimonials.php to read real quotes from published authors. 